\title{
New Notions for Discrete Topology
}

\author{
Gilles Bertrand \\ Laboratoire A2S, ESIEE Cité Descartes B.P. 99 \\ 93162 Noisy-Le-Grand Cedex France, e-mail: bertrand@esiee.fr
}

\begin{abstract}
Some new notions based on orders and discrete topology are introduced. We investigate the notions of unipolar and free points, we propose some discrete definitions for homotopy and a generalization of the notion of simple point.
\end{abstract}

Keywords: discrete topology, order, homotopy, simple point

\section{Introduction}

Several approaches have been proposed for the study of topological properties of binary digital images:

- the digital topology approach introduced by A. Rosenfeld ([15], [11]). Elements of $\mathcal{Z}^{n}$ are linked by some adjacency relations which allow to define connectedness. There is no straightforward way to build a topology which corresponds to this notion of connectedness $([6],[2])$.

- the connected ordered topological space (COTS) approach introduced by E. Khalimsky ([7], [8], [10]). The smallest neighborhood of each point of $\mathcal{Z}^{n}$ differs from one point to another. This allows to recover the structure of a topology.

- the complex cellular approach. An object is seen as a structure consisting of elements of different dimensions called cells. As noticed by V. Kovalevsky [12], it is also possible, with this approach, to recover the structure of a topology.

The topology which is used in the last two approaches is an Alexandroff or a discrete topology [1] which is a topology such that every intersection of open sets is open. There is a link between Alexandroff topologies and (partially) ordered sets (orders), i.e., sets on which a reflexive, antisymmetric, and transitive binary relation $\preceq$ is defined. Recall that a topological space $E$ is $T_{0}$-separable if, for any two elements of $E$, there exists an open subset $O$ such that exactly one of the two elements is in $O$. We may define an order on the points of any $T_{0}$-separable topological space. This order is such that $x \preceq y$ if and only if $x$ is in the closure of $\{y\}$. Conversely, an order determines an Alexandroff topology: a set $O$ is open for this topology if and only if $x \in O$ and $x \preceq y$ implies $y \in O$.

This paper is the first of a series which investigate some new notions for discrete topology. We introduce the notion of unipolar point which may be seen as the simplest "inessential" element. We propose some discrete definitions for homotopy, and show there is a link between retraction and unipolar points. Then we present a generalization of the classical notion of simple point. 


\section{Basic notions}

If $X$ is a set, $\mathcal{P}(X)$ denotes the set composed of all subsets of $X$, if $S$ is a subset of $X, \bar{S}$ denotes the complement of $S$ in $X$. If $S$ is a subset of $T$, we write $S \subseteq T$, the notation $S \subset T$ means $S \subseteq T$ and $S \neq T$. If $\gamma$ is a map from $\mathcal{P}(X)$ to $\mathcal{P}(X)$, the dual of $\gamma$ is the map $* \gamma$ from $\mathcal{P}(X)$ to $\mathcal{P}(X)$ such that, for each $S \subseteq X$, $* \gamma(S)=\overline{\gamma(\bar{S})}$. Let $\theta$ be a binary relation on $X$, i.e., a subset of $X \times X$. The inverse of $\theta$ is the binary relation $\{(x, y) \in X \times X ;(y, x) \in \theta\}$. We also denote $\theta$ the map from $X$ to $\mathcal{P}(X)$ such that, for each $x$ of $X, \theta(x)=\{y \in X,(x, y) \in \theta\}$. We define $\theta^{\square}$ as the binary relation $\theta^{\square}=\theta \backslash\{(x, x) ; x \in X\}$. Let $x_{0}$ and $x_{k}$ be two elements of $X$. A $\theta$-path from $x_{0}$ to $x_{k}$ is a sequence $x_{0}, x_{1}, \ldots, x_{k}$ of elements of $X$ such that $x_{i} \in \theta\left(x_{i-1}\right)$, with $i=1, \ldots, k$; the number $k$ is the length of the path, $x_{0}$ is its origin.

\subsection{Orders and discrete topology}

An order is a pair $|X|=(X, \alpha)$ where $X$ is a set and $\alpha$ is a reflexive, antisymmetric, and transitive binary relation on $X$. An element of $X$ is also called a point. The set $\alpha(x)$ is called the $\alpha$-adherence of $x$, if $y \in \alpha(x)$ we say that $y$ is $\alpha$-adherent to $x$. We also denote $\alpha$ the map from $\mathcal{P}(X)$ to $\mathcal{P}(X)$ such that, for each subset $S$ of $X, \alpha(S)=\cup\{\alpha(x) ; x \in S\}, \alpha(S)$ is called the $\alpha$-closure of $S$, $* \alpha(S)$ is called the $\alpha$-interior of $S$. A subset $S$ of $X$ is $\alpha$-closed if $S=\alpha(S), S$ is $\alpha$-open if $S=* \alpha(S)$.

Let $(X, \alpha)$ be an order. We denote $\beta$ the inverse of $\alpha$. Sometimes, in order to recall this notation, we also call an order any triplet $(X, \alpha, \beta)$ where $(X, \alpha)$ is an order as defined above and $\beta$ is the inverse of $\alpha$. The dual of the order $(X, \alpha, \beta)$ is the order $(X, \beta, \alpha)$. Note that $* \alpha(S)=\{x \in S ; \beta(x) \subseteq S\}$, and $* \beta(S)=\{x \in S ; \alpha(x) \subseteq S\}$.

The set $\mathcal{O}_{\alpha}$ composed of all $\alpha$-open subsets of $X$ satisfies the conditions for the family of open subsets of a topology, the same result holds for the set $\mathcal{O}_{\beta}$ composed of all $\beta$-open subsets of $X$; we denote respectively $\mathcal{T}_{\alpha}=\left(X, \mathcal{O}_{\alpha}\right)$ and $\mathcal{T}_{\beta}=\left(X, \mathcal{O}_{\beta}\right)$ these two topologies. These topologies are Alexandroff or discrete topologies [1], i.e., topologies such that every intersection of open sets is open. The closed subsets for $\mathcal{T}_{\alpha}$ (resp. $\mathcal{T}_{\beta}$ ) are the $\alpha$-closed (resp. $\beta$-closed) subsets as defined above. The $\alpha$-adherence (resp. $\beta$-adherence) of a point $x$ is the smallest neighborhood for the topology $\mathcal{T}_{\beta}\left(\operatorname{resp} . \mathcal{T}_{\alpha}\right)$.

When $(X, \alpha)$ and $\left(X^{\prime}, \alpha^{\prime}\right)$ are orders, a map $f$ from $X$ to $X^{\prime}$ is order preserving or isotone if $y \in \alpha(x)$ implies $f(y) \in \alpha^{\prime}[f(x)]$. We say that $f$ is an isomorphism from $(X, \alpha)$ to $\left(X^{\prime}, \alpha^{\prime}\right)$, if $f$ is a bijection so that $y \in \alpha(y)$ if and only if $f(y) \in \alpha^{\prime}[f(x)]$, for all $x, y$ in $X$.

Let $(X, \alpha, \beta),\left(X^{\prime}, \alpha^{\prime}, \beta^{\prime}\right)$ be two orders and let $f$ be an isotone map from $X$ to $X^{\prime}$. Let us consider an element $U^{\prime} \in \mathcal{O}_{\alpha^{\prime}}$. Let $x$ be a point of $f^{-1}\left(U^{\prime}\right)$, that is $f(x) \in U^{\prime}$, and let $y \in \beta(x)$. We have $f(y) \in \beta^{\prime}[f(x)]$, hence $f(y) \in U^{\prime}$ and $y \in f^{-1}\left(U^{\prime}\right)$. Thus, $f^{-1}\left(U^{\prime}\right) \in \mathcal{O}_{\alpha}$. So, the inverse of any $\alpha^{\prime}$-open set is an $\alpha$-open set: any isotone map is continuous. The converse may be easily verified: 
If $(X, \alpha)$ and $\left(X^{\prime}, \alpha^{\prime}\right)$ are orders, a map $f$ from $X$ to $X^{\prime}$ is isotone if and only if $f$ is continuous with respect to $\mathcal{T}_{\alpha}$ and $\mathcal{T}_{\alpha^{\prime}}$.

If $(X, \alpha)$ is an order and $S$ is a subset of $X$, the order relative to $S$ is the order $|S|=(S, \alpha \cap(S \times S))$; we denote $\left.\alpha_{S}=\alpha \cap(S \times S)\right)$. When no confusion may arise, we also denote $|S|=(S, \alpha)$.

\subsection{CF-orders}

An order $(X, \alpha, \beta)$ is countable if $X$ is countable, it is locally finite if, for each $x$ of $X,[\alpha \cup \beta](x)$ is a finite set. A $C F$-order is a countable locally finite order. If $|X|=(X, \alpha, \beta)$ is a CF-order, a path in $|X|$ is a $\theta$-path with $\theta=\alpha \cup \beta$.

It may be seen that a CF-order $(X, \alpha, \beta)$ is connected for $\mathcal{T}_{\alpha}$ (or for $\mathcal{T}_{\beta}$ ) if and only if it is path-connected, i.e, if for all $x, y$ in $X$, there is a path from $x$ to $y$.

Let $(X, \alpha)$ be a CF-order. An element $x$ such that $\alpha^{\square}(x)=\emptyset$ is said to be $\alpha$-terminal. A point $y$ is an $\alpha$-terminal of $x$ if $y$ is an $\alpha$-terminal and $y \in \alpha(x)$. If $(X, \alpha, \beta)$ is an order we define $\alpha^{\bullet}$ as the relation on $X$ such that $y \in \alpha^{\bullet}(x)$ if and only if $y \in \alpha^{\square}(x)$ and $\alpha^{\square}(x) \cap \beta^{\square}(y)=\emptyset$. The set $\alpha^{\bullet}(x)$ is called the $\alpha$-closeness of $x$, if $y \in \alpha^{\bullet}(x)$ we say that $y$ is $\alpha$-close to $x$.

Note that $\alpha^{\bullet}$ is antireflexive $\left(\forall x \in X, x \notin \alpha^{\bullet}(x)\right)$, antisymmetric, and antitransitive $\left(\forall x, y, z \in X\right.$, if $y \in \alpha^{\bullet}(x)$ and $z \in \alpha^{\bullet}(y)$, then $\left.z \notin \alpha^{\bullet}(x)\right)$. In fact, $\alpha$ is the smallest reflexive and transitive relation containing $\alpha^{\bullet}$. We also note that the relation $\beta^{\bullet}$ is the inverse of the relation $\alpha^{\bullet}$.

Let $S$ be a subset of $X$. It should be pointed out that, in general, $\alpha_{S}^{\bullet}$ is different from $\alpha^{\bullet} \cap(S \times S)$. In fact, if $x$ and $y$ are points of $S, y \in \alpha_{S}^{\bullet}$ if and only if $y \in \alpha(x)$ and $\alpha^{\square}(x) \cap \beta^{\square}(y) \subseteq \bar{S}$. Thus if $x, y \in S$ and if $y \in \alpha^{\bullet}(x)$, then $y \in \alpha_{S}^{\bullet}(x)$, the converse is, in general, not true.

Let $|X|=(X, \alpha)$ be a CF-order. The $\alpha$-decomposition of $|X|$ is the family $\mathcal{F}=\left\{X_{0}, X_{1}, \ldots, X_{k}\right\}$ such that:

i) $X_{0}$ is composed of all $\alpha$-terminals; and

ii) $X_{i}$ is composed of all $\alpha_{S}$-terminals, with $S=X \backslash \cup\left\{X_{j} ; j=0, \ldots, i-1\right\}$; and iii) $X_{k} \neq \emptyset$ and $X=\cup\left\{X_{i} ; i=0, \ldots, k\right\}$.

The $\alpha$-rank of a point $x$ is the number $r_{\alpha}(x)=i$ such that $x \in E_{i}$.

The family $\mathcal{F}$ is a disjoint family of non-empty subsets of $X$ whose union is $X$ : $\mathcal{F}$ is a partition of $X$.

We note that $r_{\alpha}(x)$ is the length of a longest $\alpha^{\square}$-path having $x$ as origin.

If $y \in \alpha(x)$ and $r_{\alpha}(y)=r_{\alpha}(x)-1$, then $y \in \alpha^{\bullet}(x)$, the converse is, in general, not true.

\section{$3 \quad$ Unipolar and free points}

We introduce the notion of unipolar point. Intuitively an unipolar point may be seen as the "simplest inessential" element of an order, in the sense that its removal does not "change the topology" of the order. The introduction of the notion of free point is motivated by the Property 4 which indicates that the 
adherence of such points has a specific structure (see [5] for a presentation of this structure).

Definition 1: Let $(X, \alpha)$ be a CF-order. We say that a point $x$ of $X$ is $\alpha$-unipolar if $\alpha^{\bullet}(x)$ consists in exactly one point.

Thus, a point $x$ is $\alpha$-unipolar if and only if there exists a point $y \in X$ such that $\alpha^{\square}(x)=\alpha(y)$.

Definition 2: Let $|X|=(X, \alpha)$ be a CF-order and let $x$ be a point of $X$. We say that $x$ is $\alpha$-free if $x$ is $\alpha$-unipolar or if there is a sequence $x_{0}, \ldots, x_{k}$ with $x_{k}=x$, such that $x_{0}$ is $\alpha$-unipolar and $x_{i}$ is $\alpha_{i}$-unipolar, with $\alpha_{i}=\alpha \cap\left(S_{i} \times S_{i}\right)$, $S_{i}=X \backslash\left\{x_{0}, \ldots, x_{i-1}\right\}, i=1, \ldots, k$. A point which is not $\alpha$-free is called an $\alpha$-link. The $\alpha$-kernel of $X$ is the subset of $X$ composed of all $\alpha$-links of $X$. Two orders $(X, \alpha)$ and $\left(X^{\prime}, \alpha^{\prime}\right)$ are said to be $\alpha$-equivalent if the orders relative to their $\alpha$-kernel are isomorphic.

If there is a unique point $y$ which is the $\alpha$-terminal of $x$, with $y \neq x$, then it may be seen that $x$ is necessarily $\alpha$-free. The converse of this property is not true.

Property 3: Let $(X, \alpha)$ be a CF-order, $f$ be an $\alpha$-unipolar point, and let $x$ be a point of $X$, with $x \neq f$. If $x$ is $\alpha$-unipolar, then $x$ is $\alpha_{S}$-unipolar for $S=X \backslash\{f\}$.

Proof: Suppose $x$ is $\alpha$-unipolar. We denote $x^{\prime}$ (resp. $f^{\prime}$ ) the unique point of $\alpha^{\bullet}(x)\left(\operatorname{resp.} \alpha^{\bullet}(f)\right)$.

i) Suppose $f \neq x^{\prime}$. Then $x^{\prime} \in \alpha_{S}^{\bullet}(x)$. Suppose there exists a point $y$, with $y \neq x^{\prime}$ and $y \in \alpha_{S}^{\bullet}(x)$; thus $\alpha_{S}^{\square}(x) \cap \beta_{S}^{\square}(y)=\emptyset$. We must have $\alpha^{\square}(x) \cap \beta^{\square}(y) \neq \emptyset$, this implies that $\alpha^{\square}(x) \cap \beta^{\square}(y)=\{f\}$, and $\alpha^{\square}(x) \cap \beta^{\square}(f)=\emptyset, f$ would belong to $\alpha^{\bullet}(x)$ which is impossible. Thus $x$ is $\alpha_{S}$-unipolar.

ii) Suppose $f=x^{\prime}$. Then it may be seen that we must have $\alpha_{S}^{\bullet}(x)=\left\{f^{\prime}\right\}$.

Note that the converse of Prop. 3 is, in general, not true: if $x$ is $\alpha_{S}$-unipolar for $S=X \backslash\{f\}$, then $x$ is not necessarily $\alpha$-unipolar.

Property 4: Let $(X, \alpha)$ be a CF-order, $f$ be an $\alpha$-unipolar point, and let $x$ be a point of $X$, with $f \neq x$. The point $x$ is $\alpha$-free if and only if $x$ is $\alpha_{S}$-free for $S=X \backslash\{f\}$.

Proof: If $x$ is $\alpha$-unipolar we are in the conditions of Prop. 3. If $x$ is $\alpha$-free and not $\alpha$-unipolar there is a sequence $x_{0}, \ldots, x_{k}$ with $x_{k}=x$, such that $x_{0}$ is $\alpha$ unipolar and $x_{i}$ is a $\alpha_{i}$-unipolar, with $\alpha_{i}=\alpha \cap\left(S_{i} \times S_{i}\right), S_{i}=X \backslash\left\{x_{0}, \ldots, x_{i-1}\right\}$, $i=1, \ldots, k$. Suppose $f \neq x_{i}$, with $i=1, \ldots, k$. By using $k$ times Prop. $3, f$ is $\alpha_{1}$-unipolar,..., $\alpha_{k}$-unipolar. Let $S=X \backslash\{f\}, S_{i}^{\prime}=S \backslash\left\{x_{0}, \ldots, x_{i-1}\right\}, \alpha_{i}^{\prime}=$ $\alpha \cap\left(S_{i}^{\prime} \times S_{i}^{\prime}\right)$. The point $x_{0}$ is $\alpha_{S}$-unipolar, $x_{i}$ and $f$ are $\alpha_{i}$-unipolar, thus $x_{i}$ is $\alpha_{i}^{\prime}$-unipolar, $i=1, \ldots, k$ (Prop. 3 ). Hence $x_{k}$ is $\alpha_{S}$-free.

If $f=x_{0}, x_{k}$ is $\alpha_{S}$-free, suppose $f=x_{i}$, for some $i=1, \ldots, k$. The point $x_{0}$ is $\alpha_{S}$-unipolar, if $1 \leq j<i, x_{j}$ is $\alpha_{j}^{\prime}$-unipolar (by the preceding analysis) and if $i<j \leq k$, trivially $x_{j}$ is $\alpha_{j}^{\prime}$-unipolar. Thus $x_{k}$ is $\alpha_{S}$-free.

On the other hand, by the very definition of $\alpha$-free points, if $x$ is $\alpha_{S}$-free, with $S=X \backslash\{f\}$, then $x$ is $\alpha$-free.

We may reformulate the Prop. 4 in the following way: if $(X, \alpha)$ is a CF-order and if $x \in X$ is $\alpha$-unipolar, then the $\alpha_{S^{-}}$-kernel for $S=X \backslash\{x\}$, is precisely the $\alpha$ - 
kernel. This indicates a way for computing the $\alpha$-kernel: we choose arbitrarily an $\alpha$-unipolar point and we remove it; we repeat this operation until idempotence.

Since, in a CF-order, connectedness is equivalent to path-connectedness, we see that, if we remove from $X$ an $\alpha$-unipolar point, we do not change the number of connected components of $X$. Thus, by induction, we have the following property:

Property 5: $A C F$-order $(X, \alpha)$ is connected if and only if the order relative to its $\alpha$-kernel is connected.

\section{Collapse and free points}

Let $E$ be a set. An (abstract) simplicial complex on $E$ is a family $\mathcal{F}$ of finite and non-empty subsets of $E$, called simplexes, such that (see [14]):

i) if $x \in E$, then $\{x\} \in \mathcal{F}$; and

ii) if $S \in \mathcal{F}$, then each non-empty subset of $S$ belongs to $\mathcal{F}$.

If $S \in \mathcal{F}$ has precisely $k+1$ elements $(k \geq 0), k$ is the dimension of $S$. If $E \neq \emptyset$ the dimension $n$ of $\mathcal{F}$ is the largest $k$ such that $\mathcal{F}$ contains an $n$-simplex. Any $T \subseteq S$, with $S, T \in \mathcal{F}$ is a face of $S$, if $T$ is a proper subset of $S, T$ is a proper face of $S$. A principal simplex of $\mathcal{F}$ is a simplex which is not a proper face of any simplex in $\mathcal{F}$. For any $S \in \mathcal{F}$, a free face of $S$ is a proper face $R$ of $S$ which is not a proper face of any simplex of $\mathcal{F}$ besides $S$. If $\mathcal{F}$ has a principal simplex $S$ which has a free face $T$, the family $\mathcal{G}=\mathcal{F} \backslash\{S, T\}$ is a simplicial complex, we say that this complex has been obtained from $\mathcal{F}$ by an elementary collapse. A sequence of elementary collapses is a collapse.

To any simplicial complex $\mathcal{F}$ on $E$ we may associate the CF-order $|X|=$ $(X, \alpha, \beta)$, with $X=\mathcal{F}$ and $\alpha=\supseteq$, thus $G \in \alpha(F)$ if $F \supseteq G$. It may be seen that a principal simplex is a $\beta$-terminal, a free face $R$ is a $\beta$-unipolar element. Nevertheless, the notion of unipolar point in an order as it has been introduced is different from the notion of free face used in simplicial complexes. In simplicial

(1)

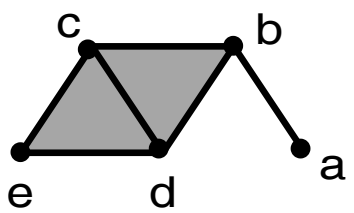

(3)

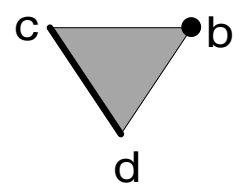

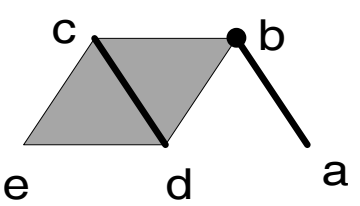

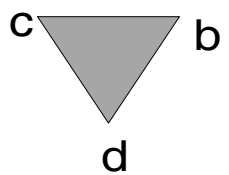

(2)

Fig. 1. Kernels of an order associated to a simplex 
complexes we cannot remove free faces in "parallel" (the resulting object would not be a simplicial complex), so it is not possible to define, for example, the equivalent of the notion of $\alpha$-kernel. In Fig. 1 (1), we give an illustration of an order $|X|$ associated to a 2D simplicial complex: we have $E=\{a, b, c, d, e\}$, $\mathcal{F}=\{\{a\},\{b\},\{c\},\{d\},\{e\},\{a, b\},\{b, c\},\{b, d\},\{c, e\},\{c, d\},\{d, e\},\{b, c, d\}$, $\{c, d, e\}\}$. An element $x$ of $\mathcal{F}$ which contains 1 (resp. 2, 3) elements of $E$ is represented by a black dot (resp. segment, triangle). The order $|X|$ contains no $\alpha$-free points. In Fig. 1 (2), the $\beta$-kernel of $|X|$ is depicted, in Fig. 1 (3) the $\alpha$ kernel of the precedent order is given, then we have the $\beta$-kernel of the precedent order (Fig $1(4)$ ) which consists in the element $\{b, c, d\}$ which is not a simplex. We see that the notion of dimension is different from the one which is often associated to orders and which corresponds to the dimension of simplicial complexes. In this framework, an element of an order is considered as a 0-dimensional entity.

\section{Some orders associated to $\mathcal{Z}^{n}$}

We give now a presentation of some orders which may be associated to $\mathcal{Z}^{n}$, these orders are equivalent to the ones obtained in the framework of connected ordered topological spaces introduced by E.D. Khalimsky [7].

Let $E$ be a set and let $E^{n}$ be the Cartesian product of $n$ copies of $E$. An element $a$ of $E^{n}$ may be seen as a map from $\{1, \ldots, n\}$ to $E, a(i)$ is the $i$-th coordinate of $a, i=1, \ldots, n$. If $S$ is a subset of $E^{n}$, the $i$-th projection of $S$ is the set $S(i)=\{a(i) ; a \in S\}, i=1, \ldots, n$.

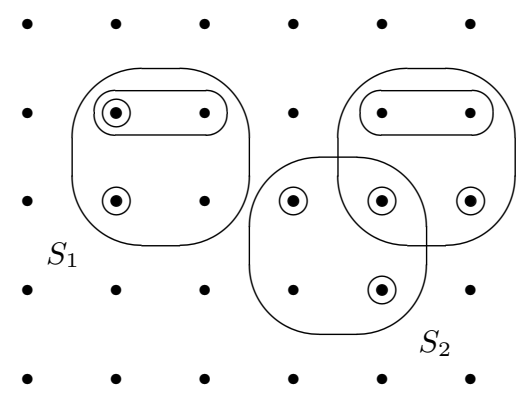

(a)

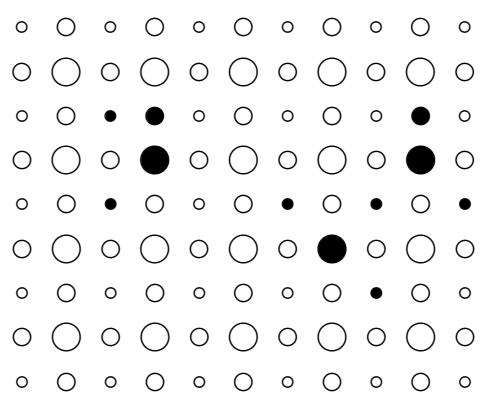

(b)

Fig. 2. An object in the set representation (a), and in the array representation (b)

Let $\mathcal{Z}$ be the set of relative integers. We consider the families of sets $H_{0}^{1}, H_{1}^{1}$, $H^{1}$ such that, $H_{0}^{1}=\{\{a\} ; a \in \mathcal{Z}\}, H_{1}^{1}=\{\{a, a+1\} ; a \in \mathcal{Z}\}, H^{1}=H_{0}^{1} \cup H_{1}^{1} . \mathrm{A}$ subset $S$ of $\mathcal{Z}^{n}$ which is the Cartesian product of exactly $m$ elements of $H_{1}^{1}$ and $(n-m)$ elements of $H_{0}^{1}$ is called a $m$-cube of $\mathcal{Z}^{n}$, we denote $H^{n}$ the set composed 
of all $m$-cubes of $\mathcal{Z}^{n}, m=0, \ldots, n$. A $m$-cube of $\mathcal{Z}^{n}$ is called a singleton if $m=0$, a unit interval if $m=1$, a unit square if $m=2$, a unit cube if $m=3$.

We denote $* \mathcal{Z}=\{a+1 / 2 ; a \in \mathcal{Z}\}$ and $\mathcal{Z}_{+}=\mathcal{Z} \cup * \mathcal{Z}$. We consider the map $\Psi_{1}$ on $H^{1}$ to $\mathcal{Z}_{+}$such that, for each $x$ of $H^{1}, \Psi_{1}(x)=a$ if $x=\{a\}, a \in \mathcal{Z}$ and $\Psi_{1}(x)=a+1 / 2$ if $x=\{a, a+1\}, a \in \mathcal{Z}$.

We also consider the map $\Psi_{n}$ on $H^{n}$ to $\mathcal{Z}_{+}^{n}$ such that, for each $x$ of $H^{n}$, the $i$-th coordinate of $\Psi_{n}(x)$ is the image of the $i$-th coordinate of $x$ under $\Psi_{1}$, i.e., $\left[\Psi_{n}(x)\right](i)=\Psi_{1}[x(i)], i=1, \ldots, n$. Note that $\Psi_{n}$ is a bijection. If $S \subset H^{n}$, $\Psi_{n}(S)=\left\{\Psi_{n}(x), x \in S\right\}$ may be seen as an array representation of $S$.

We consider the basic order associated to $\mathcal{Z}^{n}$ as the order $\left(H^{n}, \alpha\right)$, where $\alpha=\supseteq$, thus $y \in \alpha(x)$ if $x \supseteq y$. Let us examine the Fig 2 (a) where a subset $S$ of $H^{2}$ is depicted, using the usual representation for sets. The same subset $S$ is represented in Fig. 2 (b) as an array representation. We see that the subset $S$ is made of two connected components $S_{1}$ and $S_{2}$. We note that $S_{1}$ has two $\alpha$-terminals, one $\alpha$-free point, one $\beta$-terminal, three $\beta$-free points. The set $S_{1}$ is $\beta$-equivalent to an isolated point.

\section{Homotopy and deformation retract}

We propose some definitions relative to homotopy which may be seen as discrete equivalents of the classical continuous ones [14]. In order to make such a transposition, we first give few preliminary notations.

Let $|X|=(X, \alpha)$ and $\left|X^{\prime}\right|=\left(X^{\prime}, \alpha^{\prime}\right)$ be two CF-orders. The Cartesian product of the two orders $|X|$ and $\left|X^{\prime}\right|$ is the order $\left|X \times X^{\prime}\right|=\left(X \times X^{\prime}, \alpha \times \alpha^{\prime}\right)$ where $\alpha \times \alpha^{\prime}$ is the relation on $X \times X^{\prime}$ such that for each $\left(x, x^{\prime}\right) \in X \times X^{\prime}$, $\left(\alpha \times \alpha^{\prime}\right)\left(x, x^{\prime}\right)=\left\{\left(y, y^{\prime}\right) \in X \times X^{\prime} ; y \in \alpha(x)\right.$ and $\left.y^{\prime} \in \alpha^{\prime}\left(x^{\prime}\right)\right\}$. We define $I_{k}=\{\{i\},\{i, i+1\} ; i \in \mathcal{Z}$ and $0 \leq i \leq k\}$, with $k \in \mathcal{Z}, k \geq 0$. We consider the CF-order $\left|I_{k}\right|=\left(I_{k}, \supseteq\right),\left|I_{k}\right|$ is the order associated to an interval of the set of integers.

Let $|X|=(X, \alpha, \beta)$ and $\left|X^{\prime}\right|=\left(X^{\prime}, \alpha^{\prime}, \beta^{\prime}\right)$ be two CF-orders. As in the continuous framework, we could say that two isotone maps $f, g: X \rightarrow X^{\prime}$ are homotopic if there is a homotopy $h$ between them, i.e., an isotone map $h$ : $X \times I_{k} \rightarrow X^{\prime}$ with $h(x,\{0\})=f(x)$ and $h(x,\{k, k+1\})=g(x)$ for all $x \in X$, and for some $k \geq 0$. In fact $h$ is isotone if $\forall x \in X, \forall a \in I_{k}$, if $y \in \alpha(x)$ and $a \supseteq b$, we have $h(y, b) \in \alpha^{\prime}[h(x, a)]$. Let us denote $h_{i}=h(x,\{i\})$, and $h_{i+1 / 2}=h(x,\{i, i+1\}), i=0, \ldots, k$.

i) If $a=\{i\}$, with $i \geq 0$, then we have $b=\{i\}$. The condition is reduced to $h_{i}(y) \in \alpha^{\prime}\left[h_{i}(x)\right]$, we get the isotone condition for $h_{i}$.

ii) If $a=\{i, i+1\}$ with $i \geq 0$, then we have $b=\{i\}, b=\{i+1\}$, or $b=$ $\{i, i+1\}$. If $b=\{i, i+1\}$ we get the isotone condition for $h_{i+1 / 2}$. It remains the two conditions $h_{i}(y) \in \alpha^{\prime}\left[h_{i+1 / 2}(x)\right]$, and $h_{i+1}(y) \in \alpha^{\prime}\left[h_{i+1 / 2}(x)\right]$ which are equivalent to $h_{i+1 / 2}(x) \in \beta^{\prime}\left[h_{i}(y)\right]$, and $h_{i+1}(y) \in \alpha^{\prime}\left[h_{i+1 / 2}(x)\right]$.

This leads to the following definitions:

Definition 10: Let $|X|=(X, \alpha, \beta),\left|X^{\prime}\right|=\left(X^{\prime}, \alpha^{\prime}, \beta^{\prime}\right)$ be two CF-orders and let $f$ and $g$ be two isotone maps from $X$ to $X^{\prime}$. We say that $g$ is $\alpha$-homotopic 
to $f$ if $\forall y \in \alpha(x), g(y) \in \alpha^{\prime}[f(x)]$. We say that $f$ and $g$ are homotopic if there is a homotopy between them, i.e., a sequence of isotone maps from $X$ to $X^{\prime}$ : $h_{0}, \ldots, h_{k}$, with $h_{0}=f, h_{k}=g$, and such that $h_{i}$ is $\alpha$ - or $\beta$-homotopic to $h_{i-1}$, with $i=1, \ldots, k$. In this case we write $f \simeq g$.

Definition 11: Let $|X|=(X, \alpha, \beta),\left|X^{\prime}\right|=\left(X^{\prime}, \alpha^{\prime}, \beta^{\prime}\right)$ be two CF-orders. An isotone map $f$ from $X$ to $X^{\prime}$ is called a homotopy equivalence between $X$ and $X^{\prime}$ if it possesses a homotopy inverse, i.e., an isotone map $g$ from $X^{\prime}$ to $X$ with $g \circ f \simeq I d_{X}$ and $f \circ g \simeq I d_{X^{\prime}}$. In this case we say that $X$ and $X^{\prime}$ are homotopy equivalent. A CF-order is contractible if it is homotopy equivalent to an order which consists solely in one point.

It may be seen that a CF-order is contractible if and only if there is a constant map $c: X \rightarrow\{a\}$, with $a \in X$, such that $c$ and $I d_{X}$ are homotopic.

Definition 12: Let $|X|$ be a CF-order and let $S \subseteq X$. The subset $S$ is a retract of $X$ if there is a retraction, i.e. an isotone map from $X$ to $S$ such that $r(x)=x$ for each $x \in S$. If a retraction $r$ is homotopic to the identity as a map from $X$ to $X$, then $r$ is called a deformation retraction and $S$ is called a deformation retract of $X$. If this homotopy $h_{0}, \ldots, h_{k}$ between $r$ and the identity can be chosen so that $h_{i}(x)=x$ for all $x \in S$ and all $i$ with $0 \leq i \leq k, S$ is called a strong deformation retract of $X$.

Definition 13: Let $|X|$ be a CF-order, $S \subseteq X$ be a retract of $X$ and $r$ be the corresponding retraction. If $r$ is $\alpha$-homotopic to the identity as a map from $X$ to $X$, i.e., if $\forall x \in X, \forall y \in \alpha(x), r(y) \in \alpha(x)$, then $r$ is called an $\alpha$-deformation retraction and $S$ is called an $\alpha$-deformation retract of $X$.

Let $S_{0}, \ldots, S_{k}$ be a sequence of subsets of $X$, with $S_{0}=X, S_{k}=S$, and let $r_{0}, \ldots, r_{k}, r_{0}=I d_{X}, r_{k}=I d_{S}$, be a sequence of maps such that $r_{i}$ is an $\alpha$ - or a $\beta$-deformation retract on $S_{i}, S_{i}$ being the image of $S_{i-1}$ under $r_{i-1}$. Then it may be seen that $r_{i} \circ \ldots \circ r_{0}$ is homotopic to $r_{i-1} \circ \ldots \circ r_{0}$, therefore $S$ is a (strong) deformation retract of $X$.

We can associate to each $\alpha$-unipolar point $a$ an elementary $\alpha$-deformation retraction. Let $\alpha^{\bullet}(a)=\{b\}$ : we can consider the map $r$ with $r(a)=b$ and $\forall x \in X \backslash\{a\}, r(x)=x$. In fact, we also have:

Property 14: Let $|X|=(X, \alpha)$ be a CF-order and let $r$ be an $\alpha$-deformation retraction. If $r(x) \neq x$, then $x$ is an $\alpha$-free point.

Proof: We have $\forall x \in X, \forall y \in \alpha(x), r(y) \in \alpha[r(x)]$ and $r(y) \in \alpha(x)$. Let $x$ be a point with $r(x) \neq x$, we denote $y=r(x)$, we have $y \in \alpha^{\square}(x)$. The $\alpha$-rank of $x$ cannot be 0 . If the $\alpha$-rank of $x$ is 1 , we easily see that $x$ is necessarily $\alpha$-unipolar. Thus the property is true for all points $x$ with $r_{\alpha}(x) \leq 1$. Suppose the property is true for all points $x$ such that $r_{\alpha}(x) \leq(i-1)$. Let $x$ with $r_{\alpha}(x)=i$. Let us consider the set $Y=\alpha^{\square}(x) \backslash \alpha(y)$. By isotony of $r$, all points $z$ in $Y$ are such that $r(z) \in \alpha(y)$, thus $r(z) \neq z$. All these points have an $\alpha$-rank lower than $x$, by hypothesis, they are $\alpha$-free. We have $\alpha_{S}^{\bullet}(x)=\{y\}$, with $S=X \backslash Y$. Let us denote $Z$ the set of points which appear in the sequences corresponding to the definition of free points (Def. 2) and relative to the points of $Y$. It may be seen that we have $\alpha_{T}^{\bullet}(x)=\left\{y^{\prime}\right\}$, with $T=X \backslash Z$ and for some $y^{\prime} \in \alpha(y)$. Thus the point $x$ is $\alpha_{T}$-unipolar. 


\section{Simple points}

We propose now a notion of simple point based on contractible subsets.

Definition 15: Let $|X|=(X, \alpha)$ be a CF-order. A point $x$ of $X$ is $\alpha$-simple for $|X|$ if $\alpha^{\square}(x)$ is contractible.

Let $|X|=(X, \alpha)$ and $\left|X^{\prime}\right|=\left(X^{\prime}, \alpha^{\prime}\right)$ be two CF-orders. We say that $\left|X^{\prime}\right|$ is lower $\sigma$-equivalent to $|X|$, or that $|X|$ is upper $\sigma$-equivalent to $|X|^{\prime}$, if there is a sequence $X_{0}, \ldots, X_{k}$, with $X_{0}=X$, such that $X_{i}$ is obtained from $X_{i-1}$ by removing an $\alpha$ - or a $\beta$-simple point for $\left|X_{i-1}\right|, i=1, \ldots, k$, and such that $\left|X_{k}\right|$ is isomorphic to $\left|X^{\prime}\right|$. We say that $|X|$ and $\left|X^{\prime}\right|$ are $\sigma$-equivalent, if there is a sequence $\left|X_{0}\right|, \ldots,\left|X_{k}\right|$, with $\left|X_{0}\right|=|X|,\left|X_{k}\right|=\left|X^{\prime}\right|$, such that $\left|X_{i}\right|$ is lower or upper $\sigma$-equivalent to $\left|X_{i-1}\right|, i=1, \ldots, k$.

Let us consider Fig. 3 (a) where an order is depicted. Black points and segments constitute the elements of the order $\left|X_{1}\right|$, as usual we consider that $y \in \alpha(x)$ if $x \supseteq y$. The only difference between the order $X_{2}$ of Fig. 3 (b) and $X_{1}$ is an element $s$ which is represented by a square. We see that $\alpha^{\square}(s)$ is contractible: there is a sequence of $\alpha$ - and $\beta$-unipolar points which reduces $\alpha^{\square}$ to a single point; so $X_{2}$ is upper $\sigma$-equivalent to $X_{1}$. We note that all elements inside $s$ are $\beta$-simple except the four corners and that the order $X_{3}$ (Fig. 3 (c)) is lower $\sigma$-equivalent to $X_{2}, X_{4}$ (Fig. $3(\mathrm{~d})$ ) is upper $\sigma$-equivalent to $X_{3}$, we see that we cannot remove the square $s$ since $\alpha^{\square}(s)$ is not contractible. The order $X_{5}$ (Fig. $3(\mathrm{e})$ ) is upper $\sigma$-equivalent to $X_{3}$ and we can remove $s$ which leads to $X_{6}$ (Fig. 3 (f)). We note that this notion of equivalence allows to consider objects with different cardinality and thus it takes into account a difficult problem in discrete spaces. We also see that the intersection of several curves may be "moved" with

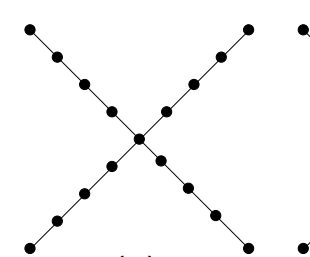

(a)

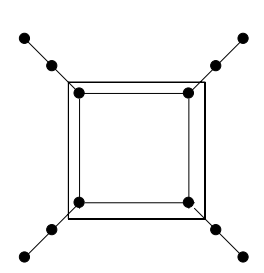

(d)

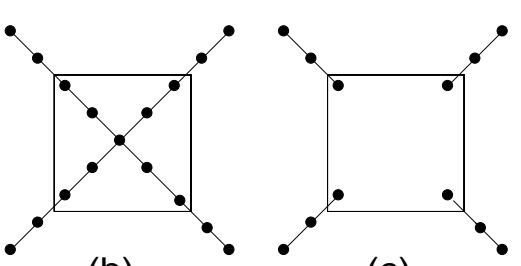

(b)

(c)

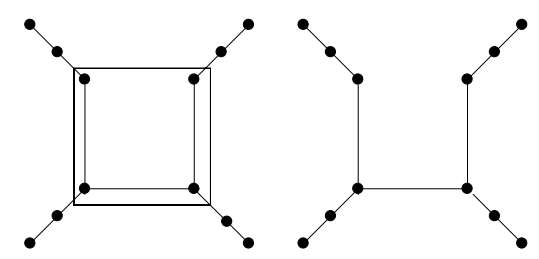

(e) (f)

Fig. 3. Illustration for simple points 
this notion of equivalence. Having this last example in mind, it is also possible to check that a "dunce hat" is $\sigma$-equivalent to a point. Dunce hat is a well-known counter-example for collapse operation. The dunce hat is an object which may be realized in a three-dimensional space and which has "no holes and cavities", i.e., its homology groups are all trivial. At last it could be seen that it is possible to "simulate" the collapse operation with the notion of simple point.

Several more specific notions of simple points may be derived from the preceding definition including some previous notions of simple points ([3], [9]). Here, we propose the following specific notion.

Definition 16: Let $|X|=(X, \alpha, \beta)$ be a non-empty CF-order. The order $|X|$ is 0 -contractible if it is composed of a single point. A point $x$ is $\alpha_{n}$-simple for $|X|$ if $\left|\alpha^{\square}(x)\right|$ is $(n-1)$-contractible, $n \geq 1$. We denote $X_{\alpha n}$ the set composed of all points of $X$ which are not $\alpha_{n}$-simple for $|X|$. The order $|X|$ is $n$-contractible, $n>0$, if there is a sequence $X^{0}, \ldots, X^{k}$, with $X^{0}=X, X^{i}=X_{\alpha n}^{i-1}$ if $i$ is odd, $X^{i}=X_{\beta n}^{i-1}$ if $i$ is even, for $i=1, \ldots, k$, and such that $X_{k}=\{a\}, a \in X$.

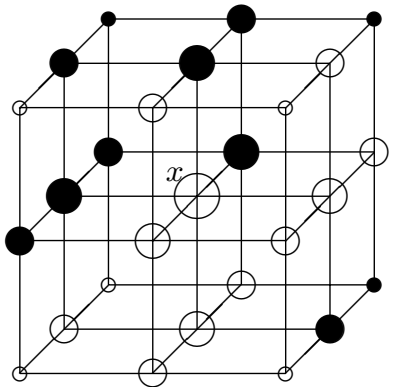

(a)

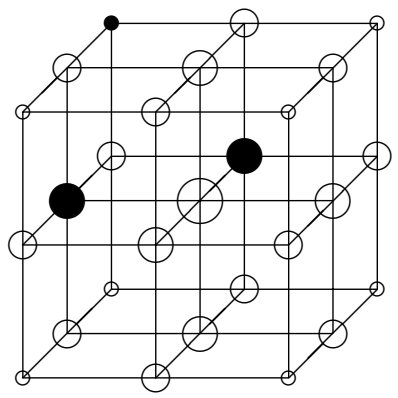

(c)

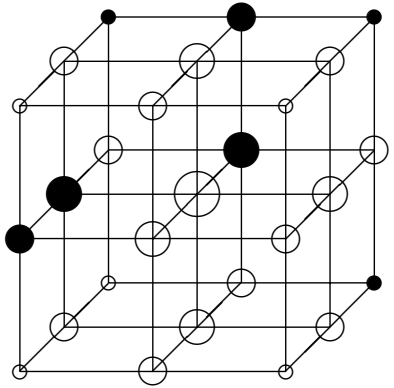

(b)

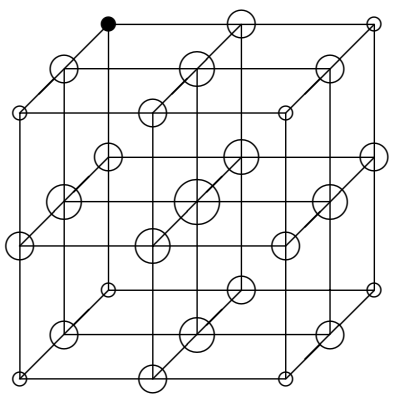

(d)

Fig. 4. Illustration of $\alpha_{3}$-simplicity

The notion of $\alpha_{1}$-simple (resp. $\alpha_{2}$-simple, $\alpha_{3}$-simple) point is appropriate for $H^{1}$ (resp. $H^{2}, H^{3}$ ), see [4]. For $n$ sufficiently large, there is the possibility 
that some patterns like the dunce hat may appear in the adherence of a point, $\alpha_{n}$-simple points cannot handle these cases. Nevertheless $\alpha_{n}$-simple points are sufficient for a lot of cases and they have some interesting features:

- The checking of an $\alpha_{n}$-simple point can be made by using polynomial algorithms;

- From this notion, thinning algorithms may be easily designed (see [13]). We may thin an object by removing alternatively $\alpha_{n}$ and $\beta_{n}$-simple points;

- $\alpha_{n}$-simple points allow to have powerful parallel algorithms. The problem of removing in parallel simple points is a difficult problem in $\mathcal{Z}^{3}$.

Fig. 4. gives an example where we test if a point $x$ is $\alpha_{3}$-simple or not for a subset $S$ of $H^{3}, x$ is the central element. We have used the array representation with the same conventions as for $H^{2}$. In Fig. 4 (a) black dots represent $\alpha^{\square}(x) \cap S$. In Fig. 4 (b), $\alpha_{2}$-simple points of $S$ have been eliminated, then $\beta_{2}$-simple points (Fig. 4 (c)), and $\alpha_{2}$-simple points (Fig. 4 (d)): $x$ is $\alpha_{3}$-simple for $S$. Note that the same Fig. 4 may also be considered as an illustration of thinning an object $S$, with $S=\alpha^{\square}(x)$.

\section{Acknowledgements}

We are pleased to thank Michel Couprie, Janusz Goldman, Maurice Nivat, and Laurent Perroton for their helpful comments.

\section{References}

1. P. Alexandroff, "Diskrete Räume", Mat. Sbornik, 2, pp. 501-518, 1937.

2. G. Bertrand, "Topologie dans les images numériques", Rapport de DEA, École Centrale des Arts et Manufactures, 1982.

3. G. Bertrand, "Simple points, topological numbers and geodesic neighborhoods in cubic grids", Pattern Rec. Letters, Vol. 15, pp. 1003-1011, 1994.

4. G. Bertrand, M. Couprie, "A model for digital topology", these proceedings.

5. G. Bertrand, in preparation.

6. J.M. Chassery, "Connectivity and consecutivity in digital pictures", Comp. Vision and Im. Proc., 9, pp. 294-300, 1979.

7. E.D. Khalimsky, "On topologies of generalized segments", Soviet Math. Doklady, 10, pp. $1508-1511,1969$.

8. E.D. Khalimsky, R. Kopperman, P. R. Meyer, "Computer graphics and connected topologies on finite ordered sets", Tology and its Applications, 36, pp. 1-17, 1990.

9. T.Y. Kong, , "Topology-preserving deletion of 1's from 2-, 3- and 4-dimensional binary images", Lect. Notes in Comp. Sciences, 1347, pp. 3-18, Springer-Verlag, 1997.

10. T. Y. Kong, R. Kopperman, P. Meyer, "A topological approach to digital topology", Discrete Comput. Geom., pp. 901-917, 1991. 13, pp. 159-166, 1989.

11. T.Y. Kong and A. Rosenfeld, "Digital topology: introduction and survey", Comp. Vision, Graphics and Image Proc., 48, pp. 357-393, 1989.

12. V. Kovalevsky, "Finite topology as applied to image analysis", Comp. Vision Graphics, and Im. Proc., 46, pp. 141-161, 1989.

13. C. Lohou, G. Bertrand, "Thinning algorithms based on discrete topology", submitted for publication.

14. J. R. Munkres, Elements of algebraic topology, Addison-Wesley, 1984.

15. A. Rosenfeld, "Digital topology", Amer. Math. Monthly, pp. 621-630, 1979. 